\title{
A Photometric Search for Dwarf Carbon Stars
}

\author{
ROBERT F. WING ${ }^{1}$ and D. JACK MacCONNELL ${ }^{2}$ \\ 1 Ohio State University, Columbus OH, U.S.A. \\ ${ }^{2}$ Computer Sciences Corp./Space Telescope Science Institute \\ Baltimore MD, U.S.A.
}

Most of the known low-luminosity carbon stars have been identified by measuring significant proper motions for stars already known to have C-type spectra. An alternative approach, which we are using in this study, is to examine the spectra of stars already known to show significant motions. This approach requires an efficient method of detecting strong $\mathrm{CN}$ or $\mathrm{C}_{2}$ bands in faint proper-motion stars.

We are using the first six filters of Wing's eight-color near-infrared narrowband photometric system to search for stars with strong $\mathrm{CN}$ absorption around $8120 \AA$ among the stars of Luyten's LHS Catalogue. The stars selected for observation are mostly red stars which lie south of $\delta=-20^{\circ}$ and lack previous classifications. Most of these stars are, of course, M dwarfs, and it is expected that the principal result of this project will be a set of accurate two-dimensional ( $\mathrm{TiO}, \mathrm{CN}$ ) classifications for several hundred $\mathrm{M}$ dwarfs not previously classified.

After three observing runs with the 1.0-m telescope at CTIO, we have "re-discovered" two previously-known $\mathrm{dC}$ stars but have not found additional examples. We have, however, found a number of stars with detectable $\mathrm{CN}$ absorption. These stars must either be giants with enormous space velocities or, more likely, dwarfs with enhanced CN. Such stars may be the result of moderate amounts of mass transferred from a former C-rich giant to a mainsequence companion. 